\title{
RECUPERAÇÃO DE PERCLORATO DE AMÔNIO A PARTIR DE RESÍDUOS DE PROPELENTE SÓLIDO COMPÓSITO
}

\author{
C. M. B. CREVELARO ${ }^{1}$, L. D. VILLAR ${ }^{2}$ e L. C. REZENDE ${ }^{2}$ \\ ${ }^{1}$ Escola Politécnica da Universidade de São Paulo, Departamento de Engenharia de Materiais \\ ${ }^{2}$ Instituto de Aeronáutica e Espaço, Divisão de Química \\ E-mail para contato: lucieneldv@iae.cta.br
}

\begin{abstract}
RESUMO - O desenvolvimento de propelente sólido compósito para aplicação na propulsão de veículos espaciais gera resíduos desse material, que são, em geral, destinados à incineração. Sendo o propelente constituído por cerca de $70 \%$ de perclorato de amônio, sal oxidante e componente energético, a lixiviação de resíduos de propelente sólido foi avaliada, em escala de laboratório, tendo-se em vista a recuperação do sal oxidante. Para tanto, a eficiência de remoção de perclorato de amônio foi determinada por medidas gravimétricas, utilizando-se delineamento fatorial de experimentos. Os resultados obtidos foram analisados aplicando-se o software Minitab ${ }^{\circledR} 16$, obtendo-se um modelo de $2^{a}$ ordem para otimização das variáveis temperatura, concentração de sólidos e velocidade de agitação. Os valores otimizados foram validados pela realização de ensaios, em triplicata, para valores de eficiência de $95 \%$.
\end{abstract}

\section{INTRODUÇÃO}

Propelentes do tipo compósito são utilizados em motores-foguete à propulsão sólida para desenvolvimento de veículos espaciais e de sistemas de defesa. Trata-se de uma mistura heterogênea entre um aglutinante polimérico e partículas sólidas, estas últimas compostas pelo oxidante, componente em maior concentração na mistura (60 a 80\%), e por um aditivo metálico, utilizado para aumentar a eficiência energética (DAVENAS, 1993; DAVENAS, 2003).

Dentre os oxidantes utilizados, destacam-se (SUTON, 1992) o perclorato de amônio, o nitrato de amônio, o perclorato de potássio e, mais recentemente, o dinitramida de amônio (RAHM et al., 2011). Os aditivos metálicos incluem alumínio em pó, boro e berílio. Dentre os aglutinantes poliméricos, são empregados (SUTON, 1992) o polibutadieno líquido hidroxilado (PBLH), o terpolímero de butadieno, poliésteres e poliéteres, com destaque para a utilização recente de polímeros energéticos, como Polímero Glicidil Azida (GAP) (SELIM et al., 2000).

Apesar do surgimento de novos materiais, a grande maioria das formulações de propelente sólido compósito (PSC) é desenvolvida empregando-se o perclorato de amônio, aglutinado em uma matriz poliuretânica formada pela reação entre um diisocianato e um poliol, sendo comum o emprego do PBLH, devido às excelentes propriedades do produto final (NAGLE et al., 2007). 


\section{9 a 22 de outubro de 2014 \\ Florianópolis/SC}

A produção de propelente sólido compósito gera resíduos deste material, devido à preparação e realização de ensaios e à estocagem de amostras-testemunha até a utilização do motor-foguete em missões de lançamento. A disposição desses resíduos tem sido conduzida por meio de incineração a céu aberto, em um processo denominado open burning incineration (SoA, 1989).

Alternativas à incineração foram propostas na década de 80, com o depósito de patentes norteamericanas. Tais documentos tinham como foco principal, estabelecer métodos para desmontagem de sistemas militares contendo PSC (SoA, 1989), meios para reduzir a sensibilidade de resíduos de propelente, permitindo sua disposição como resíduo sólido (SoA, 1988) e, finalmente, a descrição de processos ou dispositivos para recuperar perclorato de amônio (NASA, 1980) ou alumínio metálico (MORTON THIOKOL, 1987, 1988), empregados em formulações de propelente compósito.

Na década de 90, alguns estudos referentes à dessensibilização de materiais militares (BORCHERDING, 1997), incluindo-se a recuperação de explosivos (BURCH et al., 1997) foram descritos na literatura. A utilização de resíduos de propelente sólido na produção de fertilizantes foi recentemente apresentada (MEHILAL et al., 2012). Apesar dessas iniciativas, estudos sistemáticos sobre a viabilidade de recuperação e reutilização de insumos, a partir de resíduos de propelente compósito, não são comumente encontrados na literatura.

Neste contexto, o presente projeto avaliou a viabilidade do processo de solubilização (lixiviação) do perclorato de amônio a partir de amostras de propelente sólido após sua cura, considerando os fatores temperatura, concentração de sólidos e velocidade de agitação, por meio de Delineamento Fatorial de Experimentos do tipo Delineamento Composto Central Rotacional (DCCR).

\section{MATERIAIS E MÉTODOS}

\subsection{Materiais}

Resíduos de propelente compósito foram utilizados nos ensaios de lixiviação, sendo submetidos à homogeneização por cominuição manual, obtendo-se cubos com arestas de cerca de $7 \mathrm{~mm}$. Em sua composição, o resíduo de propelente continha $(\mathrm{m} / \mathrm{m})$ : perclorato de amônio (AP), 69\%; alumínio em pó, 15\%; fase aglutinante, composta de poliuretano preparado a partir da reação de PBLH e diisocianato de isoforona (IPDI), 11\%; aditivos (plastificante, antioxidante, catalisador de cura), $5 \%$.

\subsection{Ensaios de Lixiviação}

Os ensaios de lixiviação foram realizados em um reator de mistura com capacidade de $5 \mathrm{~L}$ (Láctea RT 300), com banho termostático acoplado. Para cada ensaio, foi utilizado um volume reacional de $2 \mathrm{~L}$ de água destilada. As variáveis investigadas foram temperatura, concentração de sólidos e velocidade de agitação. O planejamento fatorial foi desenvolvido considerando-se essas variáveis (fatores), combinadas a dois níveis $\left(2^{3}\right)$, tendo-se incluído seis pontos axiais e três pontos centrais (Delineamento Composto Central Rotacional, DCCR), conforme descrito por Rodrigues e Iemma (2009). A Tabela 1 apresenta os valores utilizados para os fatores estudados. 
Tabela 1 - Valores utilizados no DCCR para os fatores avaliados

\begin{tabular}{ccccccc}
\hline FATORES & FATORES & \multicolumn{5}{c}{ NÍVEIS $^{(\mathrm{a})}$} \\
\cline { 3 - 7 } & CODIFICADOS & $\mathbf{- 1 , 6 8}$ & $\mathbf{- 1}$ & $\mathbf{0}$ & $\mathbf{+ 1}$ & $\mathbf{+ 1 , 6 8}$ \\
\hline Temperatura $\left({ }^{\circ} \mathrm{C}\right)$ & $\mathrm{x}_{1}$ & 40 & 48 & 60 & 72 & 80 \\
\hline $\begin{array}{c}\text { Concentração de } \\
\text { sólidos (\%) }\end{array}$ & $\mathrm{x}_{2}$ & 5 & 9 & 15 & 21 & 25 \\
\hline $\begin{array}{c}\text { Velocidade de } \\
\text { Agitação }(\mathrm{rpm})\end{array}$ & $\mathrm{x}_{3}$ & 50 & 110 & 200 & 290 & 350 \\
\hline
\end{tabular}

(a) Níveis $(-1)$ e $(+1)$ correspondem aos pontos de vértice; níveis $(-1,68)$ e $(+1,68)$ correspondem aos pontos axiais e, nível (0) corresponde ao ponto central.

Para cada ensaio, foram retiradas alíquotas $(10 \mathrm{~mL})$ após 2, 4 e 6 horas de lixiviação, as quais foram secas em estufa a $50^{\circ} \mathrm{C}$ até massa constante.

\subsection{Cálculo de Eficiência de Recuperação de Perclorato de Amônio}

A eficiência do processo de lixiviação foi obtida por gravimetria, a partir da razão entre a massa de perclorato recuperada na alíquota $(10 \mathrm{~mL})$ e a massa de perclorato na amostra inicial (teor de 69\%), considerando-se um volume inicial de 2.000 mL (Equação 1).

$$
\text { Eficiência }_{A P}(\%)=\frac{M_{A P} \times 10}{M_{P S C} \times 0,69 \times 2000} \times 100
$$

em que, $M$ : massa; $P S C$ : propelente sólido compósito; AP: perclorato de amônio.

\subsection{Tratamento de Dados}

O tratamento de dados foi realizado utilizando-se o software Minitab ${ }^{\circledR} 16$ para ajuste do modelo preditivo em função das variáveis estudadas. A partir da análise do p-valor para nível de significância de $5 \%$, foi estabelecido o modelo preditivo de $2^{a}$ ordem. Para validação do modelo, foi fixado um rendimento de 95\%, obtendo-se os valores otimizados das variáveis. Um experimento nessas condições foi conduzido, em triplicata, e comparado com o rendimento teórico.

\section{RESULTADOS E DISCUSSÃO}

\subsection{Ensaios de Lixiviação}


Tendo-se em vista que a otimização de processos não deve ser realizada em estados de regime transitório (RODRIGUES e IEMMA, 2009), no presente trabalho, a eficiência do processo de lixiviação foi determinada ao longo do tempo, de modo a se atingir o estado de equilíbrio dinâmico, ou seja, aquele em que a eficiência de remoção do perclorato de amônio fosse máxima, para um dado conjunto de condições experimentais. Desta forma, ao longo dos ensaios de lixiviação foram retiradas amostras, em intervalos de tempos de 2, 4 e 6 horas após o início dos ensaios.

Os resultados obtidos estão apresentados na Tabela 2, em função dos fatores codificados, segundo os níveis aplicados (Tabela 1). Os ensaios apresentados compõem um Delineamento Composto Central Rotacional (DCCR), no qual os ensaios de 1 a 8 correspondem ao delineamento fatorial $2^{3}$, os ensaios 9 a 14 correspondem aos pontos axiais e, finalmente, os ensaios 15 a 17 correspondem ao ponto central.

Tabela 2 - Fatores codificados e valores de eficiência da lixiviação após 2, 4 e 6 horas

\begin{tabular}{|c|c|c|c|c|c|c|}
\hline \multirow{3}{*}{ Ensaio } & \multicolumn{3}{|c|}{ Fatores Codificados } & \multicolumn{3}{|c|}{ Eficiência $_{\mathrm{AP}}(\%)$} \\
\hline & \multirow{2}{*}{$\mathrm{x}_{1}$} & \multirow{2}{*}{$\mathrm{x}_{2}$} & \multirow{2}{*}{$\mathrm{X}_{3}$} & \multicolumn{3}{|c|}{ Tempo de lixiviação (h) } \\
\hline & & & & 2 & 4 & 6 \\
\hline 1 & -1 & -1 & -1 & 7,4 & 9,7 & 11,8 \\
\hline 2 & +1 & -1 & -1 & 12,0 & 17,8 & 21,6 \\
\hline 3 & -1 & +1 & -1 & 25,2 & 53,4 & 71,2 \\
\hline 4 & +1 & +1 & -1 & 63,5 & 73,3 & 79,0 \\
\hline 5 & -1 & -1 & +1 & 94,4 & 93,0 & 97,1 \\
\hline 6 & +1 & -1 & +1 & 92,4 & 94,4 & 96,2 \\
\hline 7 & -1 & +1 & +1 & 75,7 & 85,7 & 89,5 \\
\hline 8 & +1 & +1 & +1 & 85,8 & 93,2 & 93,4 \\
\hline 9 & $-1,68$ & 0 & 0 & 68,0 & 78,9 & 87,5 \\
\hline 10 & $+1,68$ & 0 & 0 & 80,6 & 90,1 & 93,4 \\
\hline 11 & 0 & $-1,68$ & 0 & 45,6 & 82,8 & 92,9 \\
\hline 12 & 0 & $+1,68$ & 0 & 67,7 & 76,4 & 80,9 \\
\hline 13 & 0 & 0 & $-1,68$ & 10,2 & 14,0 & 16,9 \\
\hline 14 & 0 & 0 & $+1,68$ & 87,3 & 92,9 & 94,5 \\
\hline 15 & 0 & 0 & 0 & 92,5 & 92,9 & 96,6 \\
\hline 16 & 0 & 0 & 0 & 85,6 & 90,7 & 92,9 \\
\hline 17 & 0 & 0 & 0 & 70,4 & 83,9 & 89,0 \\
\hline
\end{tabular}

A partir dos dados apresentados na Tabela 2, foram ajustadas as curvas de evolução da eficiência de remoção de AP em função do tempo (Figura 1) utilizando-se o aplicativo Origin ${ }^{\circledR} 5.0$. À exceção dos ensaios 2 e 9, todos os demais apresentaram tendência de estabilização após 6 horas de lixiviação. Sendo assim, a análise de dados utilizando o software Minitab ${ }^{\circledR} 16$ foi realizada para esses 
valores. A eficiência no equilíbrio dinâmico para os ensaios 3 e 11 foi determinada por extrapolação das curvas apresentadas, tendo-se obtidos os valores 82,7 e 99,0 \%, respectivamente.

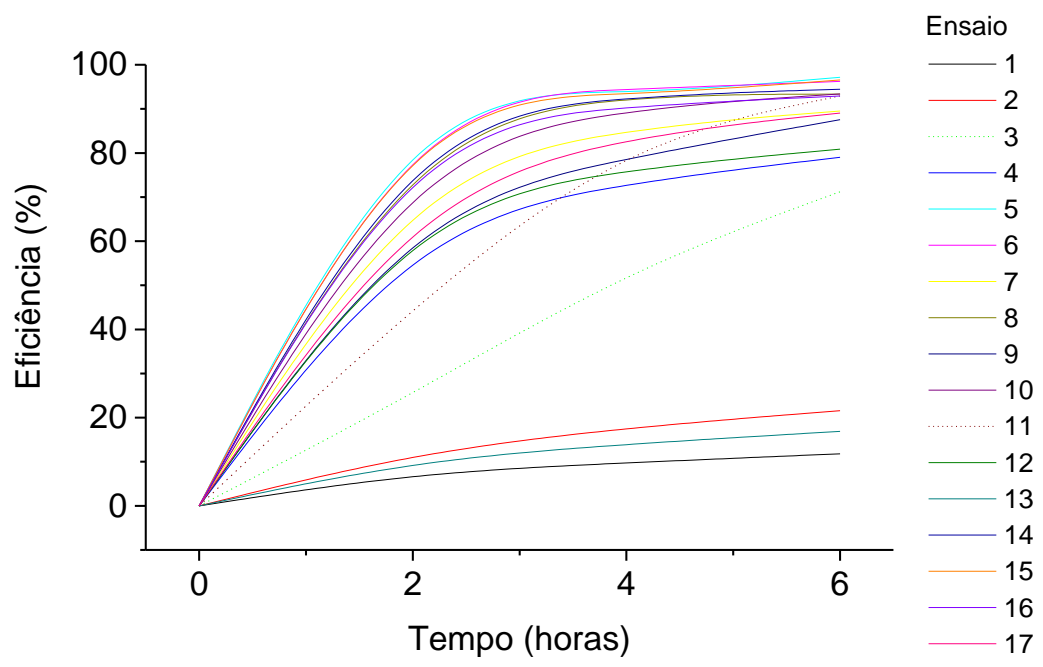

Figura 1 - Eficiência da lixiviação de perclorato de amônio em função do tempo.

\subsection{Análise de Dados}

A partir dos valores apresentados na Tabela 2, foram obtidos os coeficientes de regressão para o modelo de $2^{\mathrm{a}}$ ordem, incluindo os termos lineares, quadráticos e as interações para os fatores avaliados. A Tabela 3 apresenta os valores dos coeficientes de regressão parametrizados, conforme descrito na Tabela 1. Dados de p-valor são também apresentados para cada coeficiente. Valores de $\mathrm{p}$ maiores que 0,05 não são considerados estatisticamente significativos ao nível de significância de $5 \%$.

Tabela 3 - Coeficientes de regressão parametrizados e p-valor para modelo de $2^{\mathrm{a}}$ ordem

\begin{tabular}{ccccc}
\hline \multirow{2}{*}{ Ordem } & Fatores & $\begin{array}{c}\text { Coeficientes de Regressão } \\
\text { (parametrizados) }\end{array}$ & Erro Padrão & p-valor \\
\hline \multirow{2}{*}{ zero } & Constante & 0,93 & 0,08 & $\mathbf{0 , 0 0 0}$ \\
\hline \multirow{3}{*}{$1^{\text {a } \text { ordem }}$} & $\mathrm{x}_{1}$ & 0,02 & 0,04 & 0,740 \\
\cline { 2 - 5 } & $\mathrm{x}_{2}$ & 0,06 & 0,04 & 0,154 \\
\cline { 2 - 5 } & $\mathrm{x}_{3}$ & 0,23 & 0,03 & $\mathbf{0 , 0 0 1}$ \\
\hline \multirow{3}{*}{$2^{\text {a } \text { ordem }}$} & $\left(\mathrm{x}_{1}\right)^{2}$ & $-0,02$ & 0,04 & 0,630 \\
\cline { 2 - 5 } & $\left(\mathrm{x}_{2}\right)^{2}$ & $-0,02$ & 0,04 & 0,602 \\
\hline \multirow{3}{*}{ interação } & $\left(\mathrm{x}_{3}\right)^{2}$ & $-0,15$ & 0,04 & $\mathbf{0 , 0 1 3}$ \\
\cline { 2 - 5 } & $\mathrm{x}_{1} \cdot \mathrm{x}_{2}$ & 0,01 & 0,05 & 0,842 \\
\cline { 2 - 5 } & $\mathrm{x}_{1} \cdot \mathrm{x}_{3}$ & $-0,01$ & 0,05 & 0,942 \\
\hline & $\mathrm{x}_{2} \cdot \mathrm{x}_{3}$ & $-0,17$ & 0,05 & $\mathbf{0 , 0 1 3}$ \\
\hline
\end{tabular}


A partir da análise dos p-valores, observa-se que os coeficientes estatisticamente significativos para o modelo compreendem os termos: constante, velocidade de agitação $\left(\mathrm{x}_{3}\right)$, tanto o termo linear quanto o termo quadrático e, interação agitação-temperatura. Sendo assim, o modelo preditivo, não parametrizado, pode ser escrito como segue (Equação 2).

$$
\text { Eficiência }_{A P}=-1,42+0,075 \cdot x_{2}+0,014 \cdot x_{3}-1,70 \cdot 10^{-5} \cdot\left(x_{3}\right)^{2}-3,22 \cdot 10^{-4} \cdot x_{2} \cdot x_{3}
$$

Embora apenas os fatores velocidade de agitação e sua interação com a temperatura tenham se mostrado significativos para o modelo de predição de eficiência, as superfícies de resposta e as curvas de contorno foram elaboradas para os três fatores estudados (Figura 2), de modo a permitir avaliar graficamente o resultado estatístico.
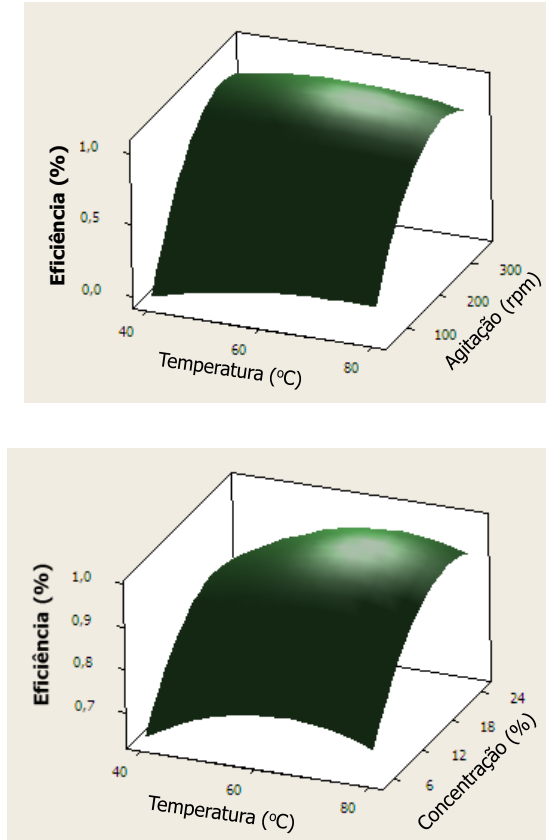

(C)

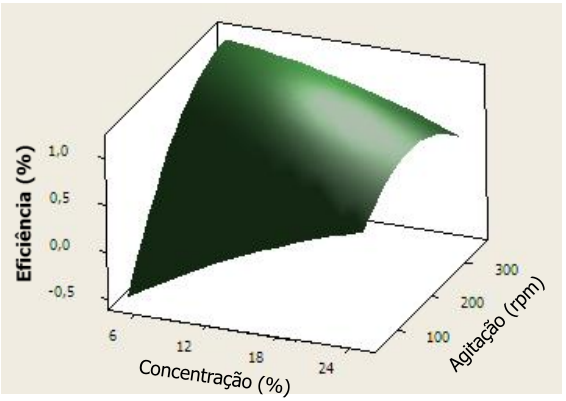

(A)

(E)

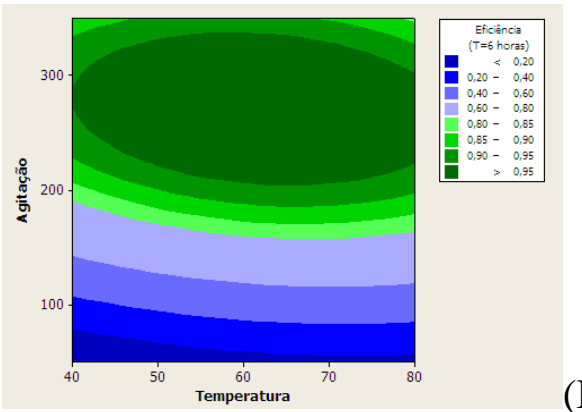

(B)
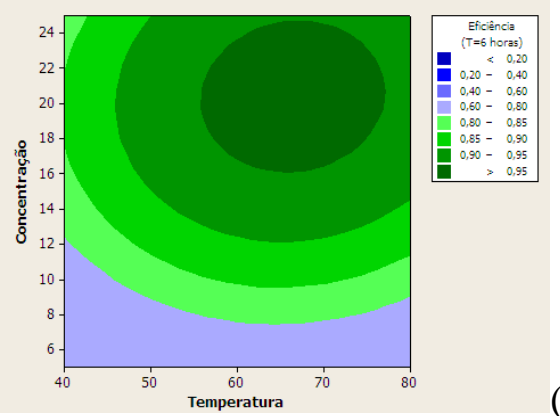

(D)

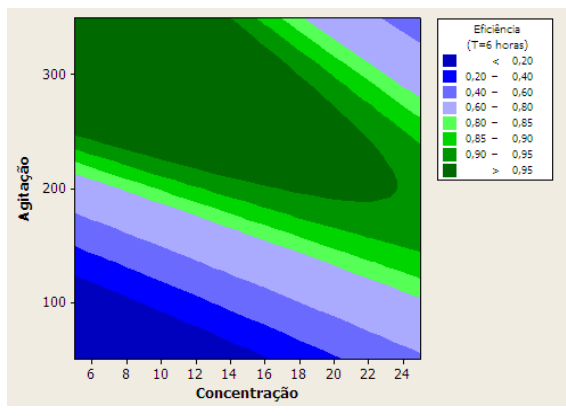

Figura 2 - Superfícies de resposta e curvas de contorno para a Eficiência (\%) de lixiviação de AP em função da temperatura e da concentração de sólidos (A) e (B), da temperatura e da velocidade de agitação (C) e (D) e, da concentração de sólidos e da velocidade de agitação (E) e (F). 
A partir da Figura 2 verifica-se que é possível obter máxima eficiência (maior que 95\%), para velocidades entre 210 e $340 \mathrm{rpm}$ para toda a faixa de temperatura estudada (Figuras $2 \mathrm{~A}$ e $2 \mathrm{~B}$ ), bem como para concentração de sólidos entre 16 e $24 \%$ para a faixa de temperatura entre 57 e $78^{\circ} \mathrm{C}$ (Figuras 2C e 2D). O comportamento da superfície E é estritamente crescente, para a faixa de valores estudada, alcançando eficiência maior que $95 \%$ para $24 \%$ de sólidos e $200 \mathrm{rpm}$ (Figuras 2E e 2F).

\subsection{Validação da Condição Otimizada}

Utilizando-se o software Minitab ${ }^{\circledR} 16$, fixou-se um rendimento de $95 \%$ tendo como limites mínimo e máximo, os valores de 90 e $100 \%$, respectivamente. A partir do modelo preditivo de $2^{\mathrm{a}}$ ordem (Equação 2), obteve-se a seguinte combinação para as variáveis estudadas: concentração de sólidos, 13,3\% e velocidade de agitação, $350 \mathrm{rpm}$. Uma vez que a lixiviação mostrou-se independente da temperatura para a faixa estudada, os ensaios de validação foram realizados na condição de $80^{\circ} \mathrm{C}$, em triplicata, obtendo-se os valores de eficiência apresentados na Tabela 4.

Tabela 4 - Resultados obtidos na condição de eficiência predita de $95 \%$

\begin{tabular}{cccccc}
\hline \multirow{2}{*}{$\begin{array}{c}\text { Tempo } \\
\text { (horas) }\end{array}$} & \multicolumn{4}{c}{ Eficiência $_{\mathrm{AP}}(\%)$} \\
\cline { 2 - 6 } & Ensaio 1 & Ensaio 2 & Ensaio 3 & Média & Erro Padrão \\
\hline 2 & 90,8 & 88,5 & 90,4 & 90 & 1 \\
\hline 4 & 95,4 & 94,3 & 93,1 & 94 & 1,1 \\
\hline 6 & 96,0 & 94,7 & 95,6 & $\mathbf{9 5 , 4}$ & $\mathbf{0 , 6}$ \\
\hline
\end{tabular}

Os resultados obtidos permitiram verificar que, após ter-se atingido o equilíbrio (6 horas), o valor final da eficiência foi de $(95,4 \pm 0,6) \%$, ou seja, coincidente com o valor teórico. Por meio desse experimento, o modelo preditivo foi validado, e a função eficiência de lixiviação de AP a partir de resíduos de propelente sólido foi maximizada para as variáveis temperatura, concentração de sólidos e velocidade de agitação.

\section{CONCLUSÃO}

A lixiviação de perclorato de amônio a partir de resíduos de propelente sólido compósito apresentou-se como um processo robusto, pouco sensível a variações de temperatura entre 40 e $80^{\circ} \mathrm{C}$. A velocidade de agitação resultou na variável de maior influência na eficiência desse processo, seguida pela concentração de sólidos. Adicionalmente, o processo de lixiviação apresentou elevado rendimento, sendo, portanto, uma excelente alternativa à disposição de resíduos de propelente.

\section{AGRADECIMENTOS}

C.M.B.C. agradece ao CNPq pela bolsa PIBIC concedida. 


\section{REFERÊNCIAS}

BORCHERDING, R. An alternative to open burning treatment of solid propellant manufacturing wastes. Waste Manag., v. 17, n. 2-3, p. 135-141, 1997.

BURCH, D.; JOHNSON, M.; SIMS, K. Value added products from reclamation of military munitions. Waste Manag., v. 17, n. 2-3, p. 159-163, 1997.

DAVENAS, A. Development of modern solid propellants. J. Propuls. Power, v. 19, n. 6, p. 11081128, 2003.

DAVENAS, A. Solid Rocket Propulsion Technology. Oxford: Pergamon Press, 1993.

MEHILAL, K.I.; DHABBE, A.K.; MANOJ, V.; SINGH, P.P.; BHATTACHARYA, B. Development of an eco-friendly method to convert life expired composite propellant into liquid fertilizer. J. Hazard. Mater., v. 205-206, p. 89-93, 2012.

MORTON THIOKOL INC. Meldon J. McIntosh. Hydraulic waste propellant macerator and method of use. US 4662893, 11 out. 1984, 5 mai. 1987.

MORTON THIOKOL INC. Ralph C. Raisor. Method of recovering powered aluminum from aluminumpolymer mixtures. US 4718955, 4 dez. 1985, 12 jan. 1988.

NAGLE, D.J.; CELINA, M.; RINTOUL L.; FREDERICKS, P.M. Infrared microspectroscopic study of the thermo-oxidative degradation of hydroxyl-terminated polybutadiene/isophorone diisocyanate polyurethane rubber. Polym. Deg. Stab., v. 92, p. 1446-1454, 2007.

NATIONAL AERONAUTICS AND SPACE ADMINISTRATION. Graham C. Shaw; Meldon J. McIntosh; North Ogden. Process for the leaching of AP from propellant. US 4198209, 29 set. 1978, 15 abr. 1980.

RAHM, M.; MALMSTROM, E.; ELDSATER, C. Design of an ammonium dinitramide compatible polymer matrix. J. Appl. Polym. Sci., v. 122, n. 1, p. 1-11, 2011.

RODRIGUES, M.I.; IEMMA, A.F. Planejamento de Experimentos e Otimização de Processo. 2 ed. Campinas: Casa do Espírito Amigo Fraternidade Fé e Amor, 2009. 358 p.

SECRETARY OF THE ARMY. David C. Sayles. Disposal of solid propellants. US 4758387, 21 abr. 1978, 19 jul. 1988.

SECRETARY OF THE ARMY. William S. Melvin; James F. Graham. Method to demilitarize extract, and recover ammonium perchlorate from composite propellants using liquid ammonia. US 4854982, 31 jan. 1989, 8 ago. 1989.

SELIM, K.; OZKAR, S.; YILMAZ, L. Thermal characterization of glycidyl azide polymer (GAP) and GAP-based binders for composite propellants. J. Appl. Polym. Sci., v. 77, p. 538-546, 2000.

SUTON, G.P. Rocket Propulsion Elements. 6. ed. New York: John Wiley \& Sons, Inc., 1992. 